\title{
BJMHR
}

British Journal of Medical and Health Research

Journal home page: www.bjmhr.com

\section{Radiologic Compilation of Spectrum of Splenic Lesions.}

Vidhyulatha Sanata ${ }^{1}$, Uma $\mathrm{M}^{2}$, Rajashekhar $\mathrm{KV}^{3}$, Sana Firdouse ${ }^{4}$.

1.Radiology consultant, Care hospitals,

2.Radiology consultant, National institute of gastrointestinal and liver diseases,

3.Radiology consultant, Vijaya diagnostics,

4.Junior Resident, Department of General Surgery, Musheerabad, Hyderabad, Telengana, 500020.India.

\section{ABSTRACT}

Spleen is a solid reticuloendothelial organ attached to stomach and kidney with ligaments.

Pathologically spleen is majorly associated with haematological disorders. The commonly encountered splenic lesions are as a result of either parasitic infections or trauma. Other causes are rare and often a neglected entity. Splenic lesions can be unifocal or multifocal. The splenic lesions can be categorized as -Congenital, normal variants, infective (acute, chronic), traumatic and mass lesions (benign or malignant). Infective and malignant lesions can be either primary originating in spleen or secondary. Metastatic focal lesions of spleen are frequently seen in malignant melanoma, renal cell carcinoma, breast carcinoma and ovarian neoplasms. Aim of our article is to review imaging findings and highlight the patterns and presentations of different splenic lesions and broaden the horizon of diagnosis from haematological pathologies to a wide varieties of existing pathologies in spleen which are often undiagnosed, missed or overlooked.

Keywords: multifocal splenic lesions, clinical imaging, inflammatory, neoplastic, vascular, traumatic. 


\section{INTRODUCTION}

Lesions in the spleen may be encountered in a variety of clinical settings ranging from asymptomatic to critically ill patients. Etiologies for multifocal splenic lesions include infectious, inflammatory, traumatic, lymphoid neoplasms, metastatic disease, vascular processes, and systemic diseases.

\section{Spectrum of Splenic lesions-}

\section{Congenital anomalies:}

1. Asplenia

2. Polysplenia

3. Splenogonadal fusion

4. Retrorenal spleen

5. Accessory spleen

6. Wandering spleen

\section{Space occupying lesions}

\section{Benign lesions}

1. Splenic abscess

2. Splenic cysts

3. Splenic epidermoid cyst

4. Splenic hemangioma

5. Splenic hamartoma

6. Splenic lymphangioma

7. Sclerosing Angiomatoid Nodular Transformation of spleen

\section{Malignant lesions}

1. Lymphoma

2. Angiosarcoma of spleen

3. Haemangiopericytoma of spleen

\section{Diffuse infiltrative systemic diseases}

1. Splenic tuberculosis

2. Splenic Granulomas

3. Splenic siderosis

4. Splenic histoplasmosis

\section{Other abnormalities}

1. Splenic rupture

2. Splenic infarct

3. Splenosis 


\section{MATERIALS AND METHOD}

Retrospectively reviewed the records and radiological studies in patients who presented with different splenic lesions and discussed the imaging features of these lesions.

\section{Congenital Splenic Lesion:}

I. Congenital Epithelial Cyst (fig:1)- incidental finding.

II. Spenunculus (Fig:2): Normal Variant

A 45yrs male patient came for HRCT chest incidental finding- splenunculus.

\section{Splenic Infections:}

I. Tuberculous Abscesses (fig:3a, 3b)

A 50 Years Male, with history of low grade fever since 2 months associated with generalized weakness and left hypochondrial pain since 1 month. History of decreased appetite and weight loss of approximately $5 \mathrm{kgs}$ in past 2 months. On evaluation diagnosed to have splenic abscess on CT.

\section{Splenic Abscess}

- $\quad 10 y \mathrm{M}$, with infective endocarditis presented with acute abdominal pain (fig:4a, 4b)

- A 42years female with history of pain abdomen, two episodes of vomitings and recurrent high grade fever not relieving on medications. She underwent evaluation and was on intermittent antibiotic therapies. On ultrasound a multicentric low density lesion was noted and ultra sounded aspirate sent for culture and sensitivity revealed-Klebsiella species growth. Antiphospholipid antibodies were found positive. (fig:4c, 4d).

\section{Hydatid Cyst Of Spleen}

A middle aged man presented chronic abdominal pain with low grade chronic fever which was relieved on medications. Ultrasonography of abdomen was suggestive of cystic lesion in spleen. (fig:5)

\section{Benign mass lesions:}

I. Splenic Lymphangiomas (fig:9)

II. Splenic Hamartoma (fig:10)

\section{Malignant Mass Lesions:}

I. Non Hodgkins Lymphoma

Middle aged male patient with history of chronic low grade fever with chills since 6 months, it relives on medications and is associated with generalized weakness and decreased appetite. he gives history of $5 \mathrm{~kg}$ weight loss in past 2 month history of small right neck swelling which increased over a period of 6 months, no association of pain or any discharging sinuses over the swelling were noted.pt was evaluated with Fine needle aspiration of the swelling which was suggestive of Lymphoproliferative 
disorder. Further evaluated with computed tomography of abdomen(fig:11) and diagnosed as a case of Non hodgkins lymphoma.

II. Splenic metastases renal cell carcinoma with splenic metastasis.

A 50yrs female presented with pain abdomen k/c/o renal mass with secondaries in liver, vertebra and focal lesion in spleen (fig:18a, 18 b)

III. Carcinoma Breast With Ovarian And Splenic Metastasis

A elderly female presented with history of progressive right breast lump associated with pain and skin changes since 6 months. History of occasional bloodish discharge from the nipple from past 2 months.(fig:19)

IV. Metastatic Adenocarcinoma (fig:20)

\section{Traumatic:}

I. Splenic Trauma Splenic Laceration

A young male was brought to emergency department with history of blunt trauma. FAST was negative. On physical examination left upper abdominal tenderness with guarding was noted. After initial stabilization contrast enhanced Computed Tomography was done which showed splenic laceration.(fig:12a, 12b).

II. Splenic Laceration With Hemoperitoneum

A 30 year old male patient was brought to emergency department with history of blunt trauma abdomen. Patient was hypotensive with severe pain abdomen. FAST was positive for free fluid in abdomen. After stabilization with intravenous fluid replacement he was contrast enhanced computed tomography which was suggestive of splenic laceration with haemoperitoneum, pt was managed with immediate surgical intervention. (fig:13a, 13b)

III. Splenic Laceration With Subcapsular Hematoma

Middle aged male presented with blunt abdomen trauma in haemodynamically stable state with complaints of pain abdomen. Diagnosed with splenic laceration with subcapsular hematoma as evidenced on his computed tomography.(fig:14a, 14b)

IV. Diaphragmatic Injury With Herniation Of Spleen (fig:17).

A 50 years male with History of blunt trauma abdomen with severe pain and in hypotension. $\mathrm{t}$

\section{Others:}

I. Vascular Multiple Splenic Artery Aneurysms (fig:6a, 6b, 6c)

A 29 years female came with history of portal hypertension on CT mesenteric angio showed multiple splenic artery aneurysms

II. splenic infarct (fig:7a, 7b) 
A 54yrs male presented with pain abdomen. when evaluated computed tomography showed Thrombotic occlusion of celiac axis with Partial thrombosis of aorta probably secondary to dissection and splenic infarction.

III. Splenic Abscess With Infarcts (fig:8)

48y M, with h/o left hypochondriac pain and on \& off low grade fever $1 \mathrm{yr}$ duration was referred for CT abdomen. Diagnosed to have splenic abscess. US guided aspiration done which revealed $25 \mathrm{ml}$ of thick hemorrhagic fluid.

At laparotomy, findings were dense adhesions around the poles of the spleen with abscesses, Final HPE Splenomegaly with infarcts and extramedullary haematopoiesis in the background of polycythaemia Vera.

IV. Splenic Rupture :

- Spontaneous Splenic Rupture (fig:15)

A 84yrs female c/o pain abdomen in left hypochondrium

- Spontaneous Splenic Rupture (fig:16)

A 20 Years male known case of Thalassemia minor with pain abdomen
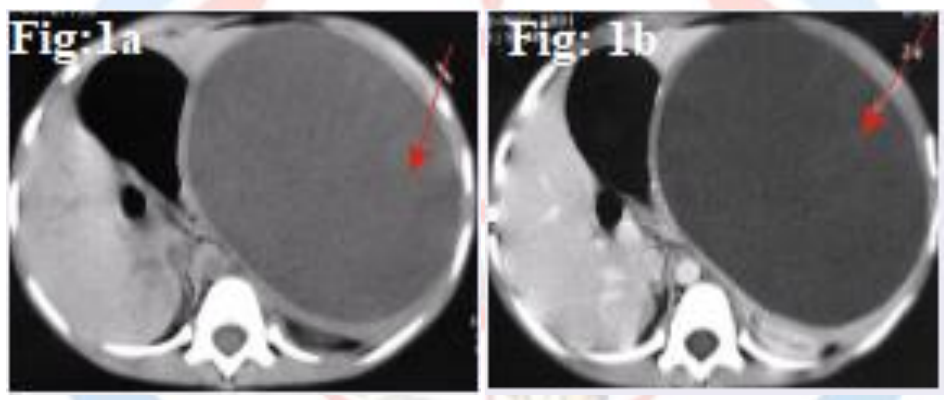

Figure:1a, 1b-Non enhancing cystic lesion in the spleen seen on computer tomography of abdomen.

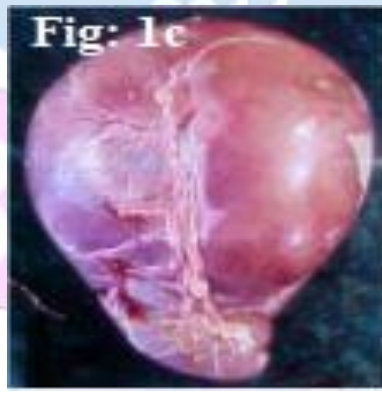

Figreu:1c- Histopathological view of congenital epithelial cyst of spleen. 

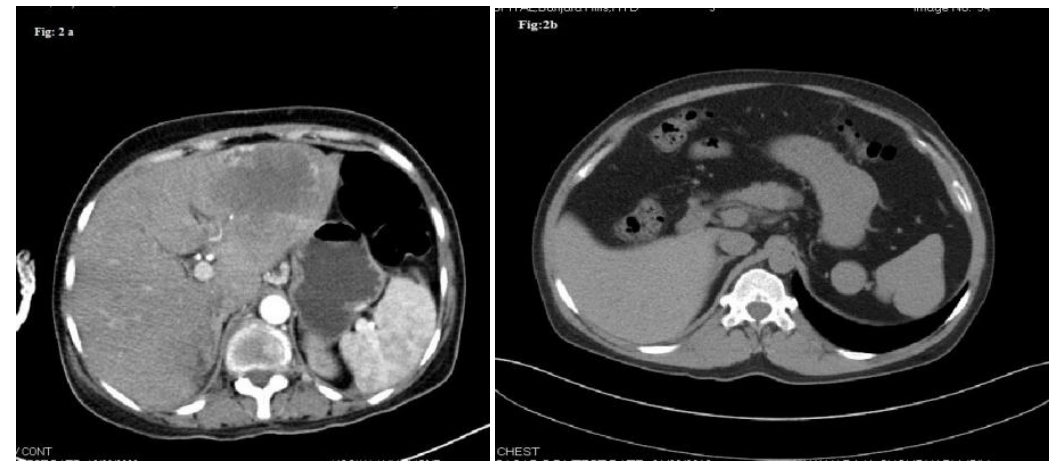

Figure: 2a, 2b- Computed Tomography -Well circumscribed ovoid isodense nodule adjacent to and seperate from spleen
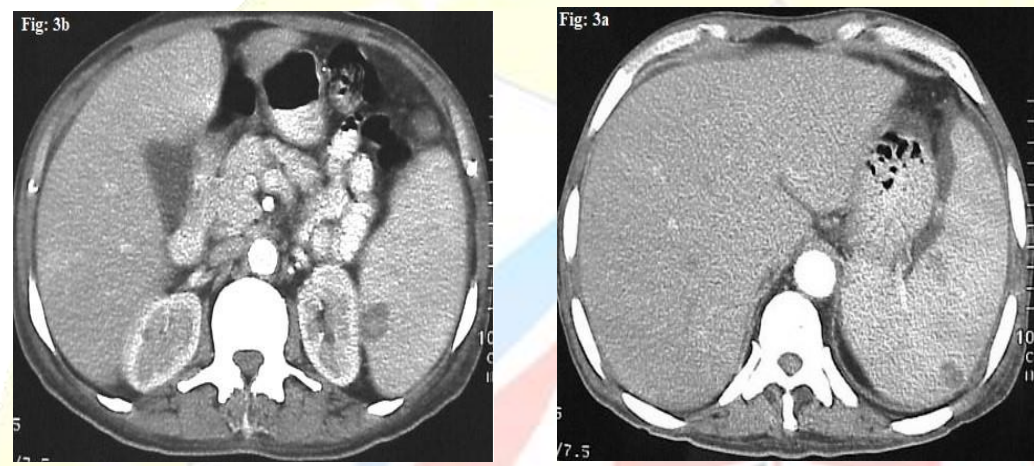

Figure:3a, 3b-Contrast enchanced computedtomography of abdomen- Multiple, welldefined, peripherally enhancing low density lesions in spleen.
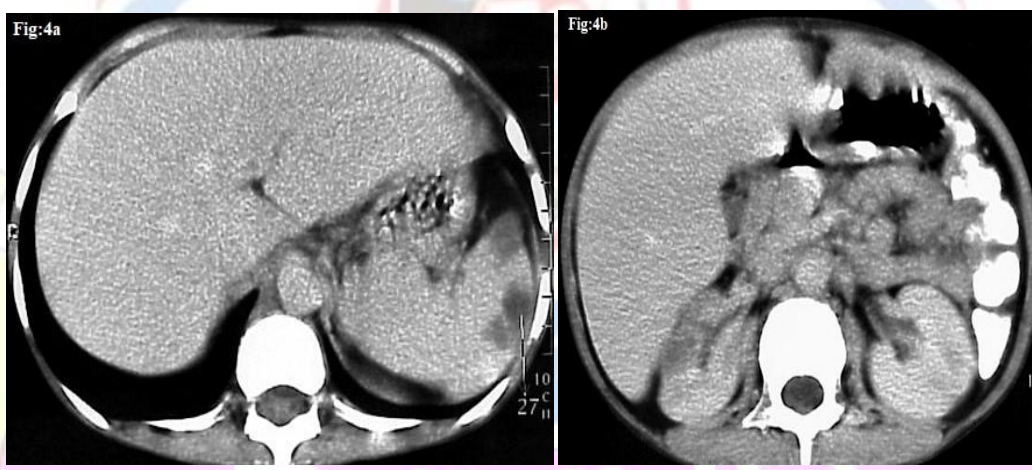

Figure: 4a, 4b- Multiple centrally low-density lesions (20-40 HU 17) seen on contrast enhanced computed tomography.

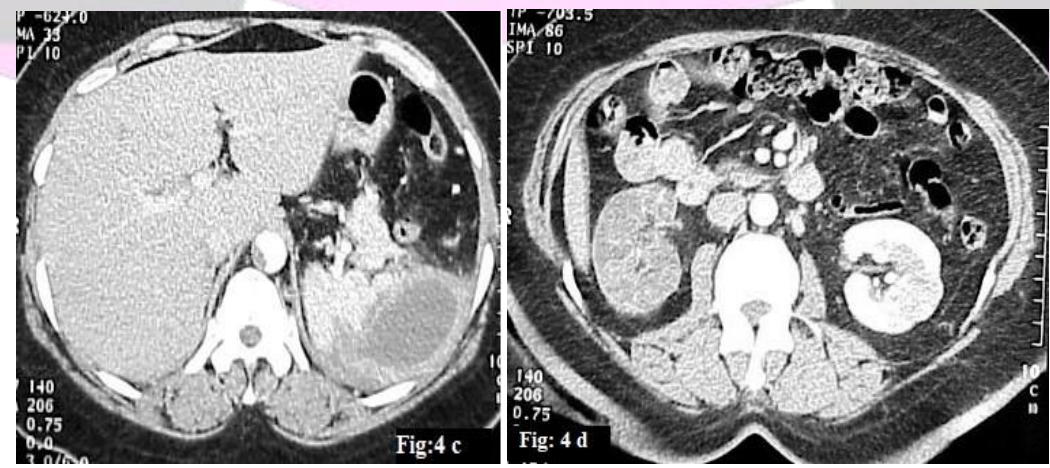


Figure: 4c, 4d- Peripherally enhancing low density lesion in spleen. Long segment eccentric thrombus in suprarenal portion of aorta can be seen on contrast enhanced computed tomography.
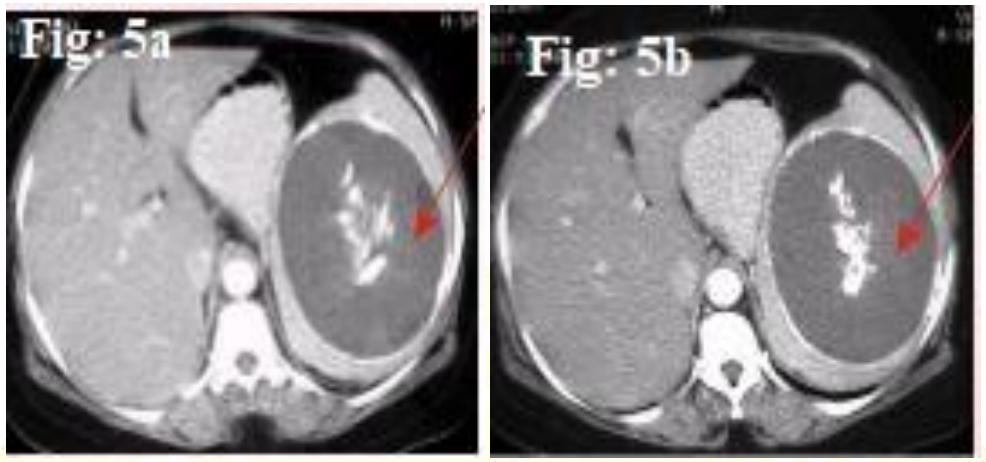

Figure:5-Contrast enhanced computed tomography of abdomen with Large cyst with wall calcifications and central calcifications.

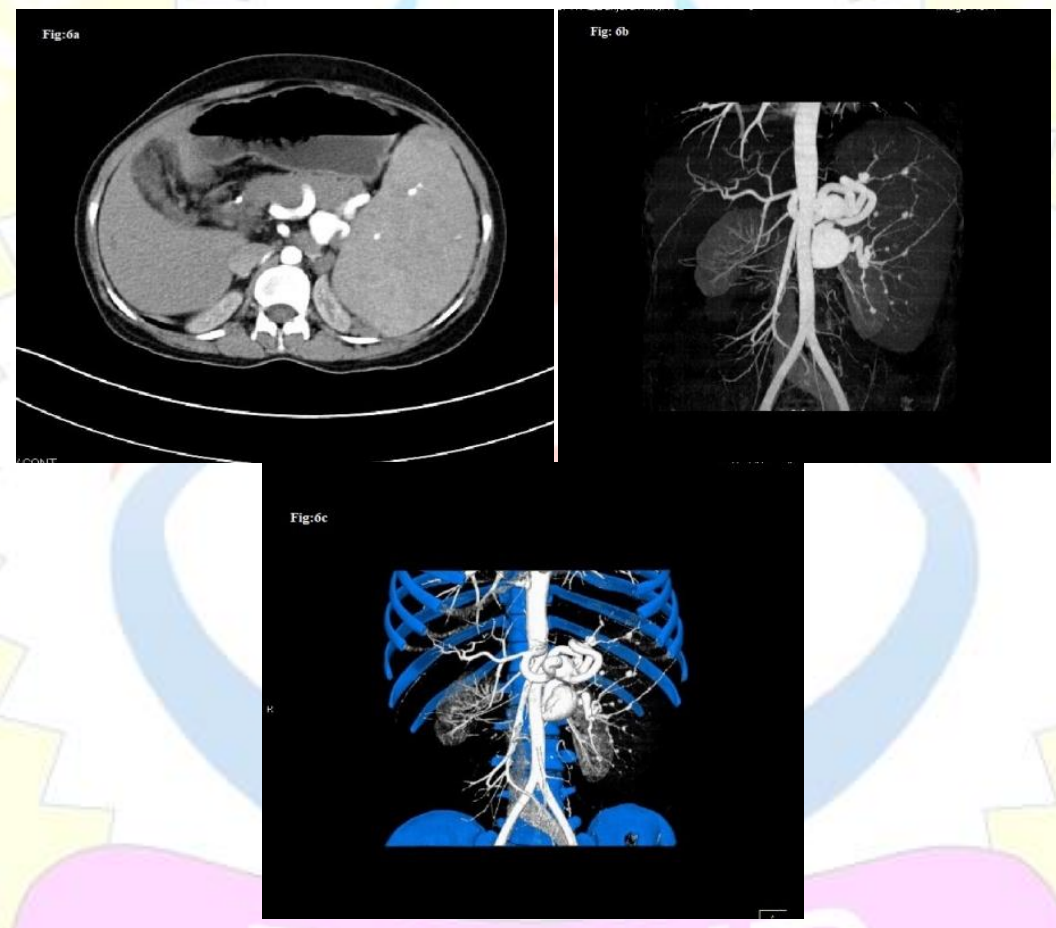

Figure: 6a, 6b, 6c- Contrast enhanced computed tomography of abdomen with angiography with Multiple splenic artery aneurysms.

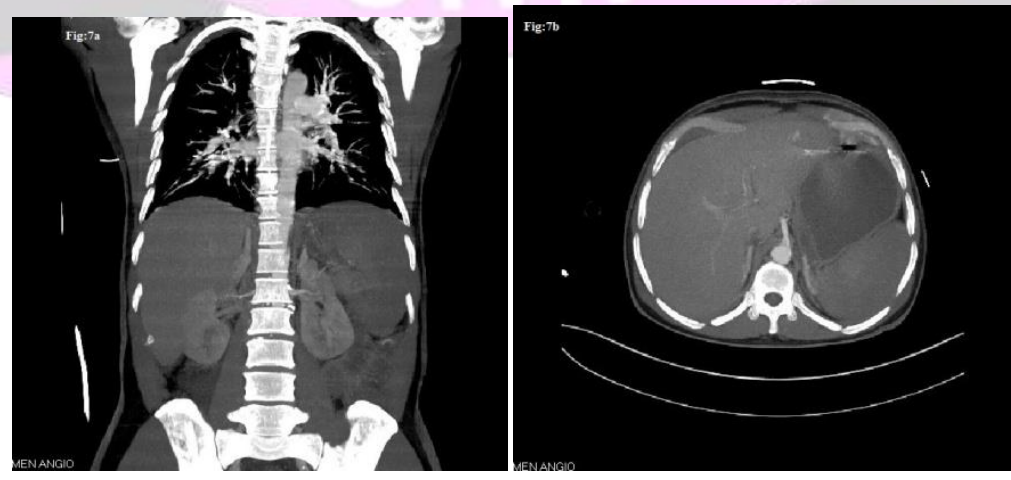

Figure: 7a, 7b-contrast enhanced computed tomography of abdomen-non enhancing wedge shaped hypodensities in spleen 

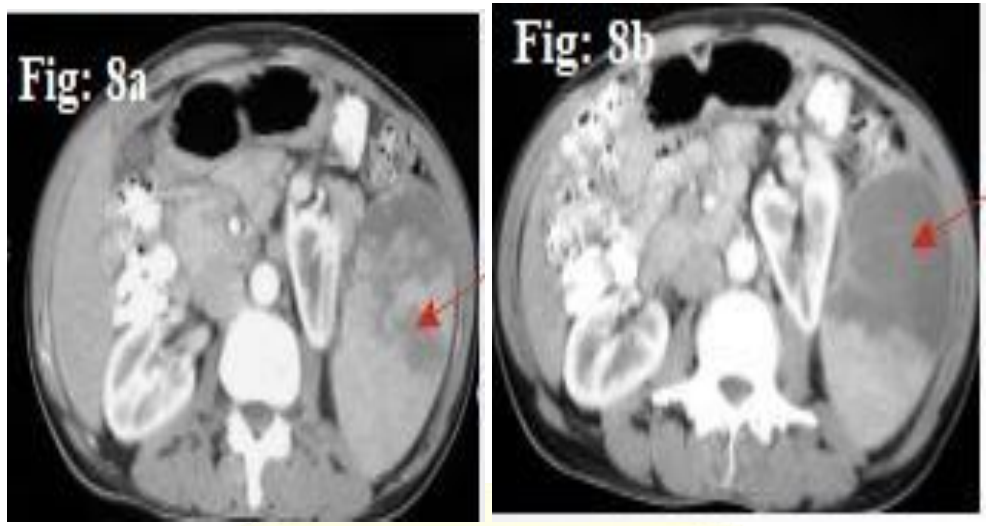

Figure: 8a, 8b-large irregular low density peripheral enhancing lesion and wedge shaped non enhancing hypodensity seen on contrast enhanching computed tomography of abdomen.
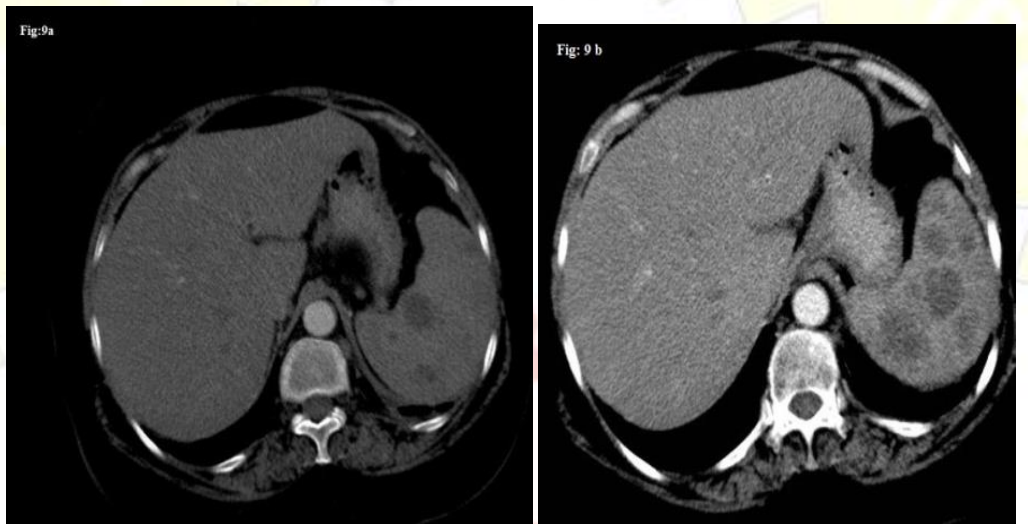

Figure: 9a, 9b-Thin walled low attenuation masses in subcapsular location with no contrast enhancement visible on contrast enhanced computed tomography of abdomen.
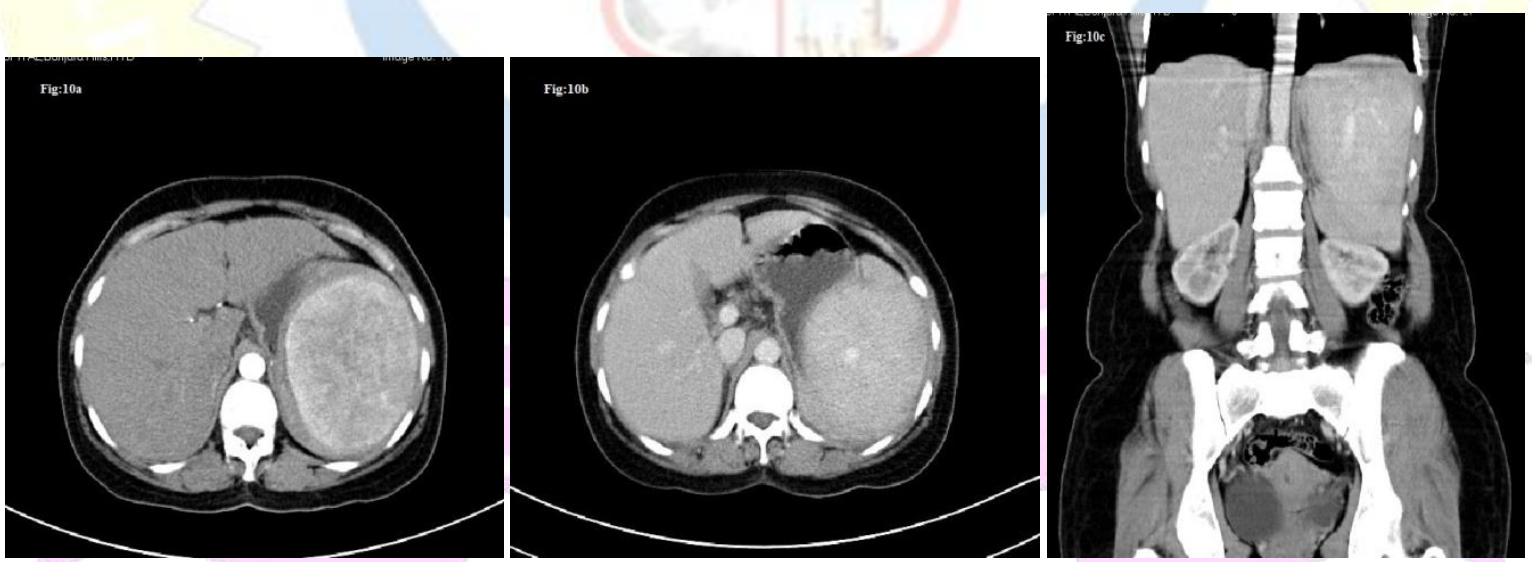

Figure: 10a, 10b, 10c- On computed tomography, isodense or hypodense solid masses and demonstrate heterogeneous contrast enhancement relative to adjacent normal parenchyma. 

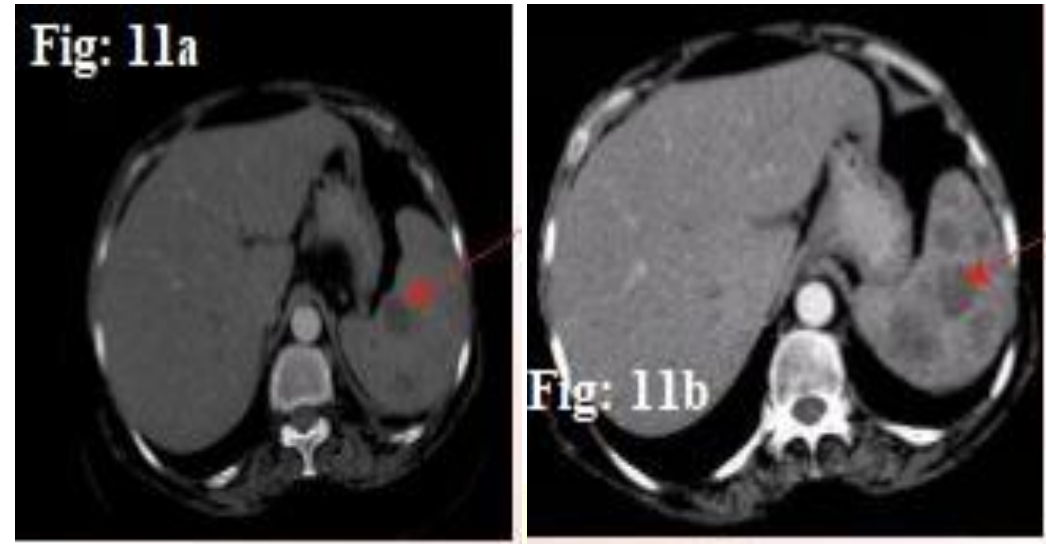

Figure: 11a, 11b-Ssplenomegaly, multimodal hypoattenuating lesions noticed on contrast enhanced computed tomography of abdomen.
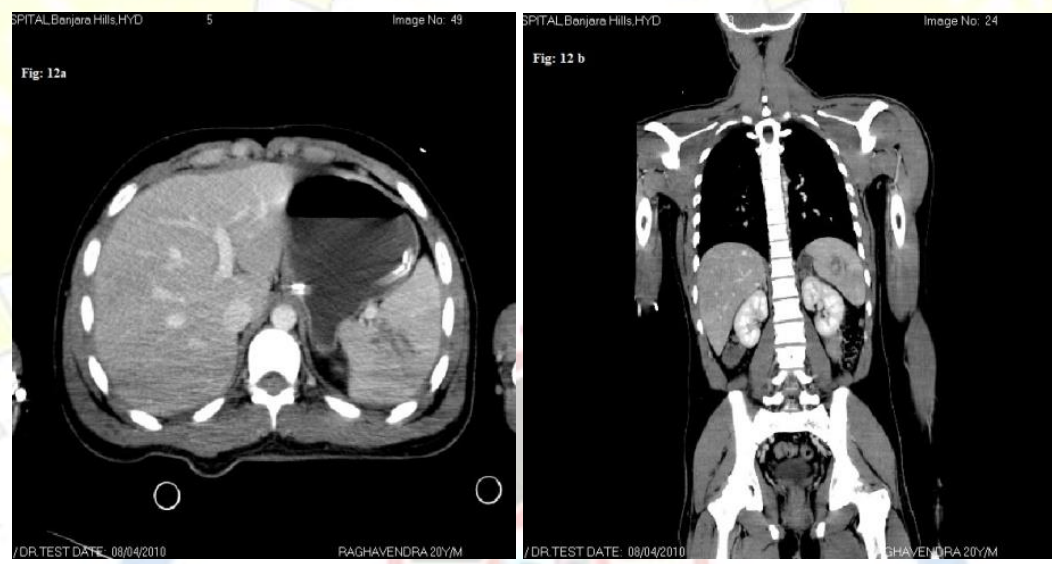

Figure: 12a, 12b- Linear or branching non enhancing hypodensities can be seen on contrast enhanced computed tomography of abdomen.

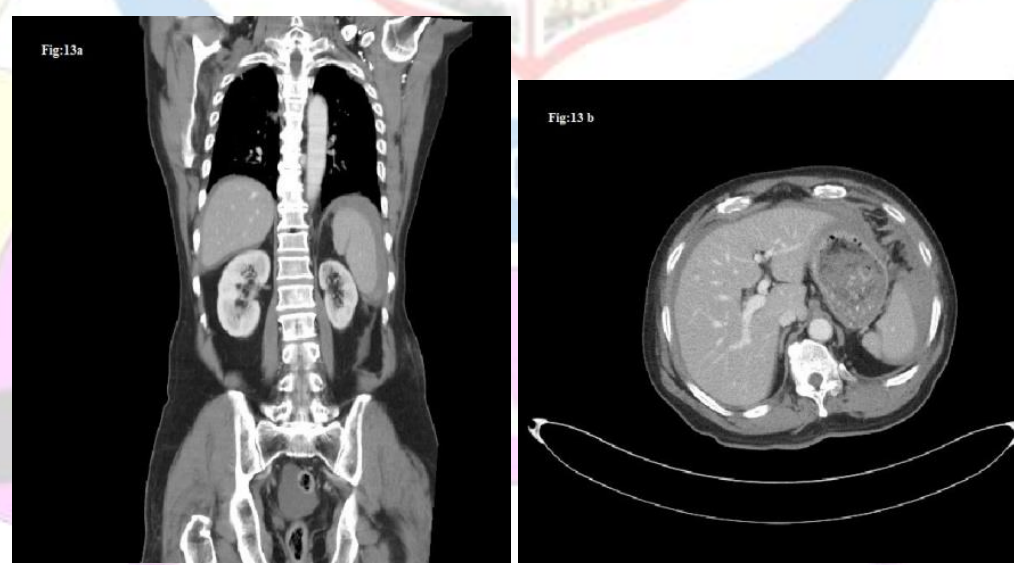

Figure: 13a, 13b- Linear non enhancing hypodensities -splenic laceration. Low density fluid in peritoneal cavity visualized on contrast enhanced computed tomography of abdomen. 

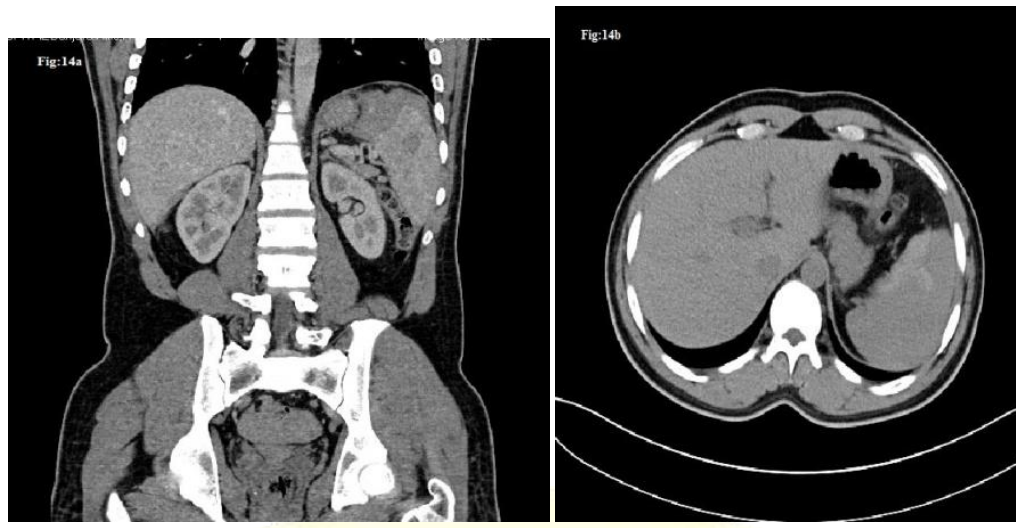

Figure: 14a, 14b- Linear non enhancing hypodensities -splenic laceration. Low-density fluid adjacent to the spleen that distorts the splenic architecture -subcapsular hematoma noted on contrast enhanced computed tomography.
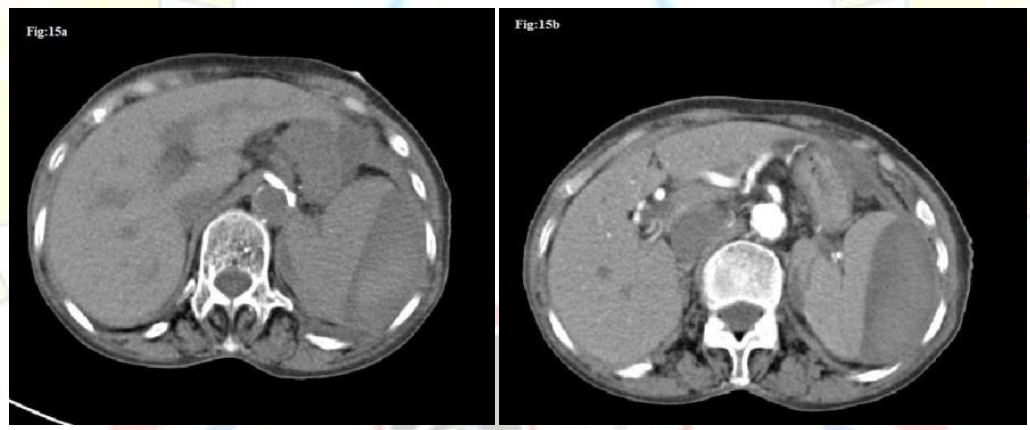

Figure: 15a, 15b- High density fluid adjacent to the spleen that distorts the splenic architecture -perisplenic hematoma seen on contrast enhanced computed tomography of abdomen.
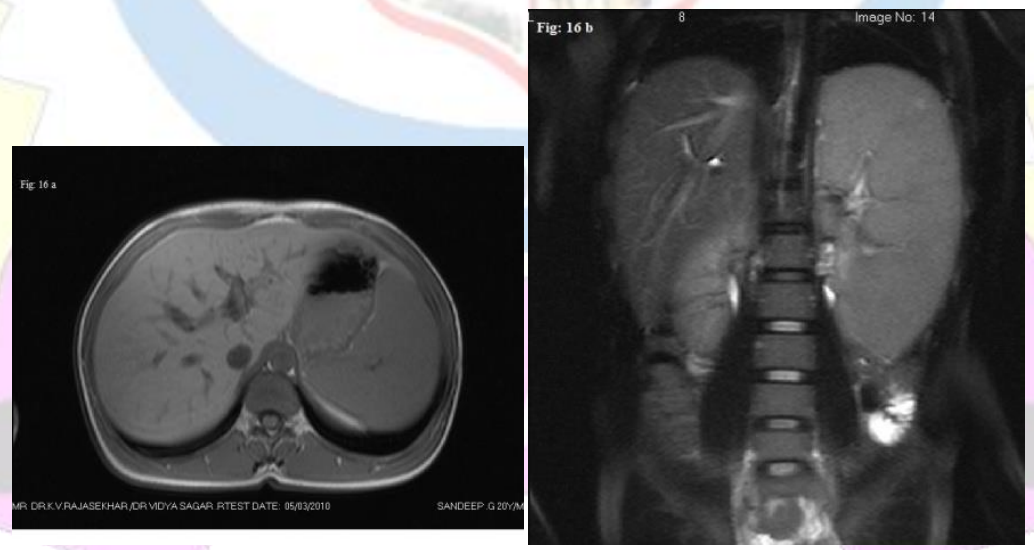

Figure: 16a, 16b- Gross spleenomegaly (M.R.I) 

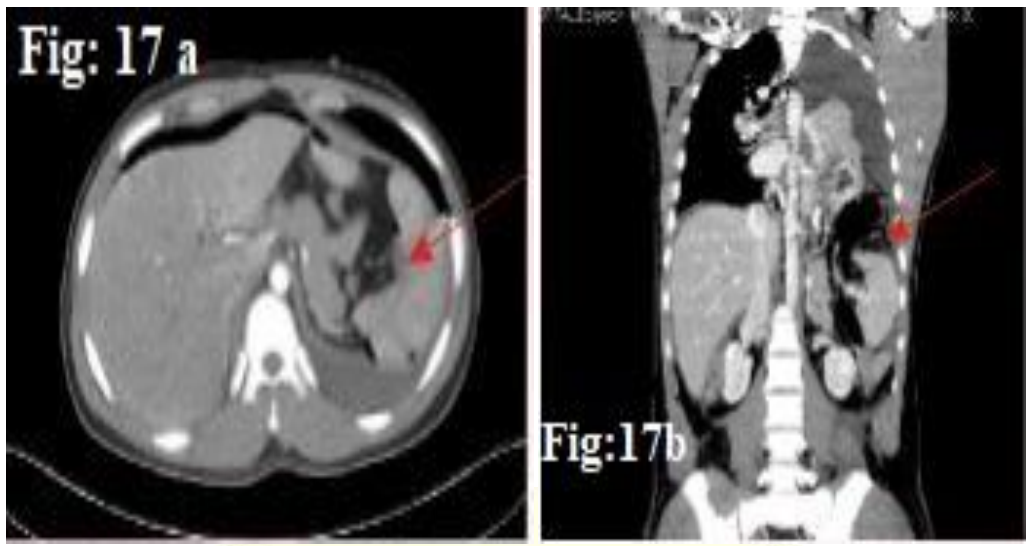

Figure:17 a, 17b-focal defect in diaphragm with herniation of spleen.

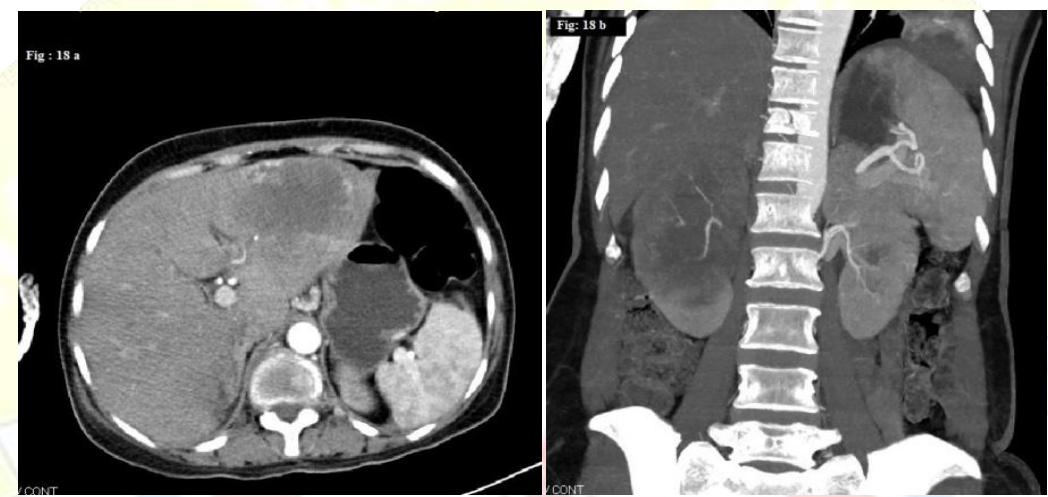

Figure: 18a, 18b- Computed tomography shows illdefined hypodense minimal peripheral enhancing lesion in spleen Large hypodense mass lesion in right kidney.

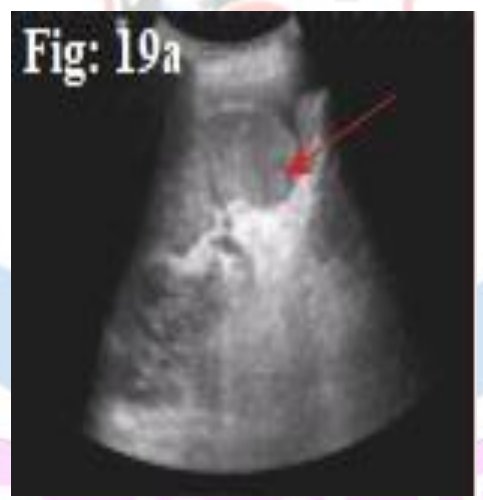

Figure: 19a-ultrasound abdomen showing well defined hypoechoic lesion in spleen.

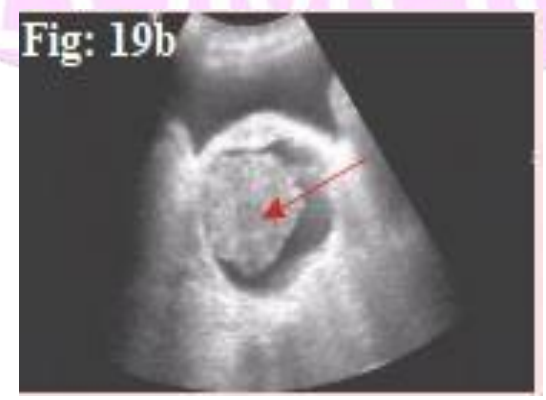

Figure:19b- Large ill defined heterogeneous mass in ovary. 


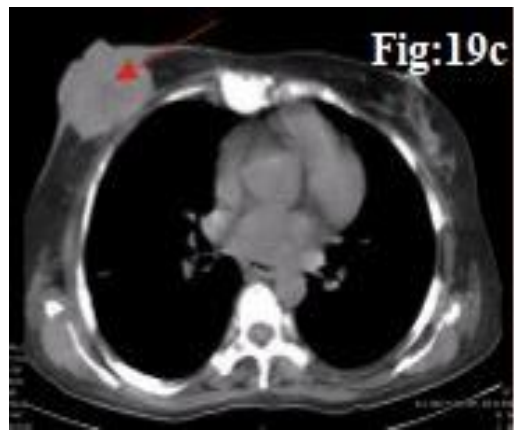

Figure:19c-Computed tomography chest showing large isodense lobulated mass in right breast infiltrating skin and subcutaneous tissue

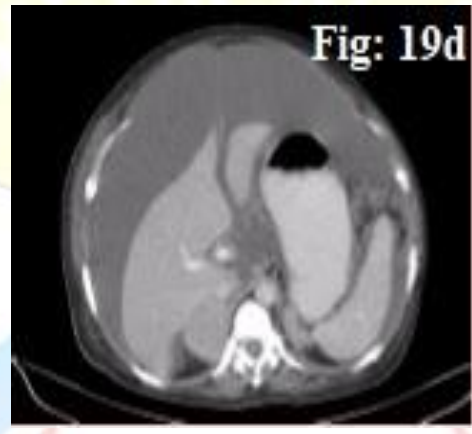

Figure: 19d-??
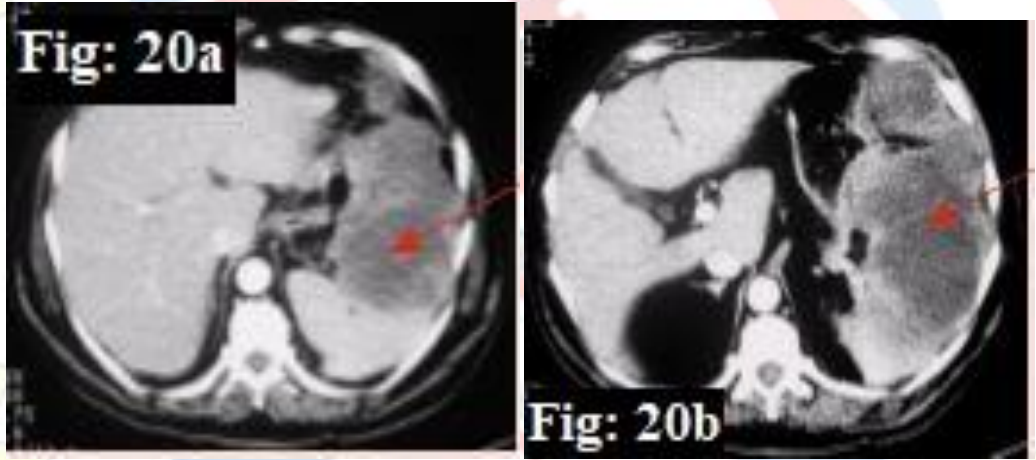

Figure: 20a, 20b-Large ill defined hypo attenuating mass in spleen

\section{RESULTS AND DISCUSSION}

There is often a overlap in the imaging appearance alone, so the clinical setting is very helpful in differential diagnosis. In immune compromised patients, multiple small splenic lesions usually represent disseminated fungal disease and micro abscesses. The spleen is a relatively rare site for metastatic disease; patients with metastatic lesions in the spleen usually have disease in other sites as well. Breast, lung, ovary, melanoma, and colon cancer are common primary tumors that metastasize to the spleen. Vascular neoplasms of the spleen represent the majority of the nonhematologic/nonlymphoid neoplasms and commonly produce multifocal lesions. Splenic infarcts may be seen with localized processes such as portal hypertension or pancreatitis, or may arise from an embolic source. we should be aware of the spectrum of processes that may involve the spleen and the clinical context in which they occur. 


\section{REFERENCE}

1. Dr Jeremy Jones, and Dr Matt A. Morgan,et al Incidental splenic lesion (an approach). -radiopedia. rID: 35298.

2. Rashid SA. Accessory Spleen: Prevalence and Multidetector CT Appearance-. Malays J Med Sci. 2014 Jul; 21(4): 18-23.

3. Saboo SS ${ }^{1}$, Krajewski KM, O'Regan KN, Giardino A, Brown JR, Ramaiya N, Jagannathan JP. Spleen in haematological malignancies: spectrum of imaging findings.2012 The British Institute of Radiology January 28, 2014

4. Kamaya $\mathrm{A}^{1}$, Weinstein S, Desser TS,Multiple lesions of the spleen: differential diagnosis of cystic and solid lesions. October 2006Volume 27, Issue 5, Pages 389403. Seminars in Ultrasound, CT and MRI.

5. Freeman $\mathrm{JL}^{1}$, Jafri SZ, Roberts JL, Mezwa DG, Shirkhoda A, CT of congenital and acquired abnormalities of the spleen..Radiographics, may 1993, volume13, issue 3.

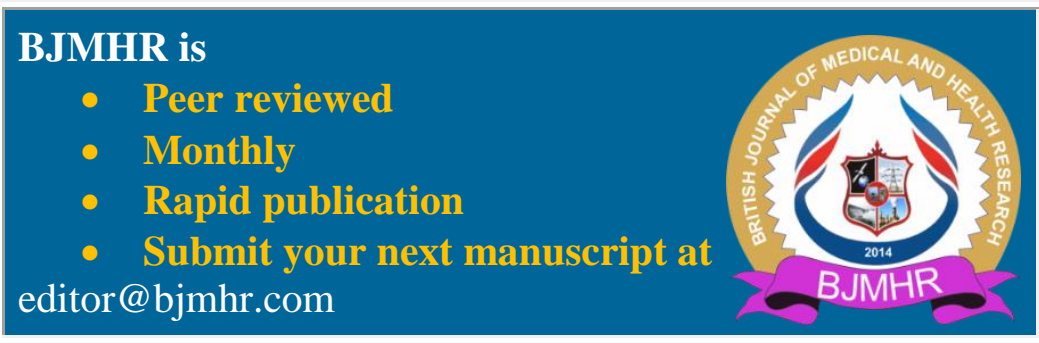

